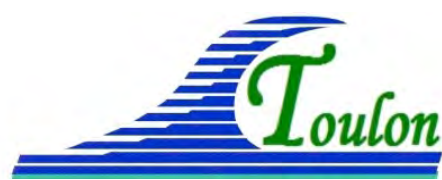

XIV èmes Journées Nationales Génie Côtier - Génie Civil

Toulon, 29 juin au $1^{\text {er }}$ juillet 2016

DOI:10.5150/jngcgc.2016.061 (C) Editions Paralia CFL

disponible en ligne - http://www.paralia.fr - available online

\title{
Quelques aspects de la déshydratation naturelle des sédiments
}

\author{
Beatriz BOULLOSA ALLARIZ ${ }^{1}$, Daniel LEVACHER ${ }^{1}$
}

1. Université de Normandie, Unicaen, Laboratoire M2C UMR6143CNRS, 24 rue des Tilleuls, 14000 Caen, France.

beatriz.boullosa-allariz@unicaen.fr ; daniel.levacher@unicaen.fr

\section{Résumé :}

Les opérations de dragage génèrent une importante quantité de sédiments qui seront considérés comme des déchets pour une gestion à terre. Le domaine du génie civil propose plusieurs applications pour leur réutilisation. Pour la plupart des applications il faut tout d'abord diminuer leur teneur en eau pour les valoriser. Le séchage naturel consiste à éliminer l'eau contenue dans les sédiments par évaporation et drainage. Cette technique est de loin la plus économique et acceptable du point de vue environnemental. Il est intéressant de rechercher, à l'aide des essais en laboratoire, des critères d'aptitude au séchage naturel des sédiments, en vue d’optimiser toute technique de déshydratation. Une synthèse d'essais de laboratoire est présentée. Ces essais ont été entrepris sur plusieurs sédiments marins et de barrage (Cherbourg, Honfleur, Isère, Durance, Rhin). Les moyens mis en œuvre sont des essais d'égouttage associés à des mesures réalisées (cohésions non drainées $\mathrm{C}_{\mathrm{u}}$ ) à l'aide d'un fall-cône et d'un scissomètre de laboratoire. Les résultats d'essais ont pu montrer que le séchage dépend du temps, des caractéristiques des sédiments et de la relation de la cohésion non drainée par rapport à la teneur en eau $\left(C_{u}-w\right)$.

Mots-clés : Sédiments, Séchage naturel, Essais d’égouttage, Fall-cône, Scissomètre, Teneur en eau, Cohésion non drainée.

\begin{abstract}
:
Dredging operations generate large amounts of sediments considered as waste. The civil engineering field offers a number of applications for their re-use. For the most of the applications at the beginning of the process, it is necessary to reduce the water content. The natural drying consists to reduce the water content in the dredging sediment by drainage and evaporation. It is the most economic and acceptable option for the environment. It appears important to optimize drying technique by the use of dewatering ability criteria. A brief recap of some tests are presented. Tests in laboratory and measurements have been be performed with fall-cone vane shear test operated in dry and drainage cells filled with marine and dam sediments (Cherbourg and Honfleur ports, Isère, Durance and Rhin rivers). Dewatering and natural drying are governed with time but also by properties of sediment and by the relation of water content and undrained shear strength $\left(C_{u^{-}}-w\right)$.
\end{abstract}


Keywords: Sediments, Natural drying, Drainage, Fall-cone, Vane shear testing, Water content, Undrained shear strength.

\section{Introduction}

Les opérations de dragage jouent un rôle important pour le développement économique, social et environnemental. Elles sont en effet nécessaires pour l'entretien des profondeurs de navigation dans les canaux, voies d'accès maritimes ou de fleuves et lors de la réalisation de travaux de génie portuaire. Elles englobent toutes les extractions de matériaux situés sur le fond d'un plan d'eau. La problématique fondamentale de ces opérations est la production d'une importante quantité de déchets qui doivent être si possible, réutilisés. En 2011, pour l'ensemble des façades maritimes françaises, un total de 262739 tonnes de sédiments ont été déposées à terre (EL FADILI \& MESSAGER, 2015).

Le génie civil propose plusieurs applications pour ces sédiments : élaboration de matériaux de remblais, routiers ou de construction, rechargement de plages et dunes ou réalisation d'ouvrages de protection ou digues sont quelques exemples. Le frein à toute valorisation matière est la teneur en eau. Il faut impérativement diminuer la teneur en eau des sédiments pour les valoriser c'est-à-dire les déshydrater. Quatre types de techniques de déshydratation peuvent être différenciés : séchage naturel, chimique, thermique et mécanique. La technique de séchage naturel est de loin la technique la plus douce, à savoir la plus économique et acceptable du point de vue environnemental. Une synthèse de résultats (DUAN, 2008 ; SANO, 2012 ; KHADRAOUI, 2012) relatifs au séchage naturel acquis en laboratoire et in situ, sur plusieurs sédiments portuaires (Cherbourg, Honfleur) et fluviaux (Isère, Durance et Rhin), est tout d'abord présentée. Elle a pour but d'aider à la détermination de critères d'aptitude au séchage et/ou déshydratation des sédiments. Cette recherche de critères est basée sur la caractérisation géotechnique et minéralogique et l'évolution de la résistance au cisaillement notamment sur l'analyse de la relation de la cohésion non drainée par rapport à la teneur en eau " $C_{u}-w$ ". L'utilisation de ces critères servira à optimiser tout type de déshydratation naturelle ou mécanisée.

\section{Matériel et méthodes}

Les essais de laboratoire concernent des essais dits d'égouttage permettant des mesures effectuées à l'aide d'un scissomètre de laboratoire et d'un fall-cône. De ces mesures, les relations "temps de déshydratation - teneur en eau" et "cohésion non drainée - teneur en eau" peuvent être établies.

Les sédiments étudiés proviennent, du port de Cherbourg (DUAN, 2008), du port d'Honfleur (KHADRAOUI, 2012) et de barrages situés en Isère, ou sur la Durance et le Rhin (SANO, 2012). 


\section{XIV èmes Journées Nationales Génie Côtier - Génie Civil \\ Toulon, 29 juin au $1^{\text {er }}$ juillet 2016}

\subsection{Essais d'égouttage}

Cet essai a été mis en place en laboratoire dans le but de déterminer le temps nécessaire pour déshydrater naturellement les sédiments d'une part et d'étudier l'évolution de la teneur en eau par rapport au temps d'autre part. Les essais d'égouttage ont été réalisés sur des sédiments disposés sur des tamis munis de géotextile à la base, pour différentes hauteurs. Les tamis ont un diamètre de $50 \mathrm{~cm}$ et un maillage variable. Le géotextile permet le drainage et évite la migration des fines.

Les paramètres de déshydratation suivis concernent : l'eau dans le sédiment " $E_{1}$ ", l'eau égouttée " $E_{2}$ " et l'eau évaporée " $E_{3}$ ". Ces quantités sont déterminées pendant la durée de l'essai.

La teneur en eau au moment des essais au scissomètre de laboratoire et fall-cône, est mesurée grâce au prélèvement de carottes miniatures (diamètre de 3mm) à différentes profondeurs, à savoir en haut, au milieu et en bas de la couche.

Pour le calcul de la masse des sédiments, en cours d'égouttage les tamis sont pesés régulièrement. Ces pesées permettent de déduire les différents volumes d'eau en fonction du temps. La durée de chaque essai dépend fortement des caractéristiques de chaque sédiment.

Les durées sont de l'ordre de 2 à 3 mois. La figure 1 montre un exemple de l'essai d'égouttage.

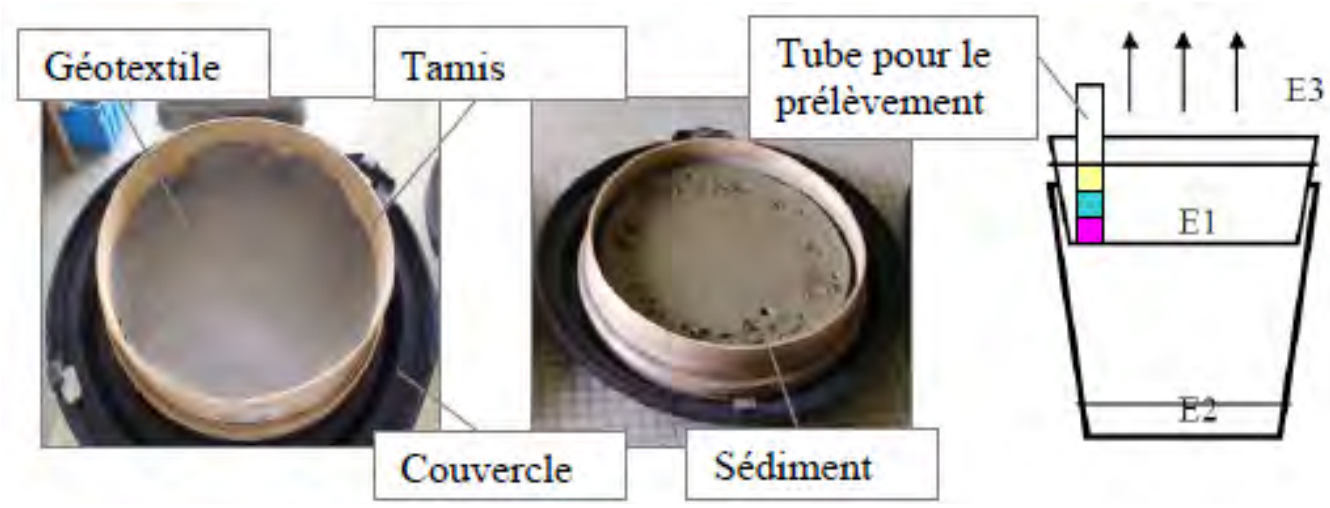

Figure 1. Principe et détails d'un essai d'égouttage

(DUAN, 2008 ; KHADRAOUI, 2012).

\subsection{Résistance au cisaillement}

La résistance au cisaillement non drainé $C_{u}$, peut être définie comme la contrainte de cisaillement maximale que les sédiments peuvent supporter. Elle joue un rôle important dans les lagunages pour la circulation d'engins dédiés au retournement d'andins, dans la transportabilité des sédiments comme dans des applications de valorisation (stabilité des terres). C'est une donnée de comportement mécanique d'un sol.

La résistance au cisaillement a été mesurée à l'aide d'essais au laboratoire: "scissomètre de laboratoire" et "fall-cône". Ces essais ont pour objectif de déterminer $C_{u}$ 


\section{Thème 6 - Gestion durable des zones littorales et estuariennes}

et de la relier à la teneur en eau et résistance au cisaillement.

\subsubsection{Essai au scissomètre de laboratoire}

Le scissomètre permet de déterminer la cohésion non drainée $C_{u}$ des sols purement cohérents (argiles, vases, tourbes). La méthode consiste à introduire verticalement une tige munie à deux pales verticales, de même largueur, dans un échantillon de sédiments. Ces pales tournent à vitesse angulaire constante jusqu'à la rupture par cisaillement du sol. La figure 2 illustre l'appareil classique utilisé. La cohésion non drainée $C_{u}$ est donnée par l'équation (1) ci-dessous :

$$
C_{u}=\frac{M}{2 \pi R^{2}(H+2 R / 3)}
$$

où $C_{u}$ est la cohésion non drainée, $M$ le moment de torsion maximal, $R$ le rayon des pales et $H$ la hauteur des pales.

\subsubsection{Essai au fall-cône}

L'essai au fall-cône ou pénétromètre à cône tombant, propose une méthode pour déterminer ou estimer la cohésion non drainée $C_{u}$ des sols en surface, relativement simple. La procédure à suivre consiste à laisser tomber un cône métallique sur la surface supérieure du sédiment et à mesurer l'enfoncement provoqué par la chute de l'ensemble tige-cône après 5 secondes de stabilisation sans vitesse initiale.

L'équation (2) ci-dessous, donne la formule à utiliser pour le calcul de cette cohésion non drainée $C_{u}$ :

$$
C_{u}=k \frac{Q}{h^{2}}
$$

où $C_{u}$ est la cohésion non drainée, $k$ une constante qui dépend des caractéristiques du cône, Q la masse du cône, et $h$ l'enfoncement du cône.

La figure 2 montre à la fois le scissomètre de laboratoire (à gauche) et le fall-cône (à droite).
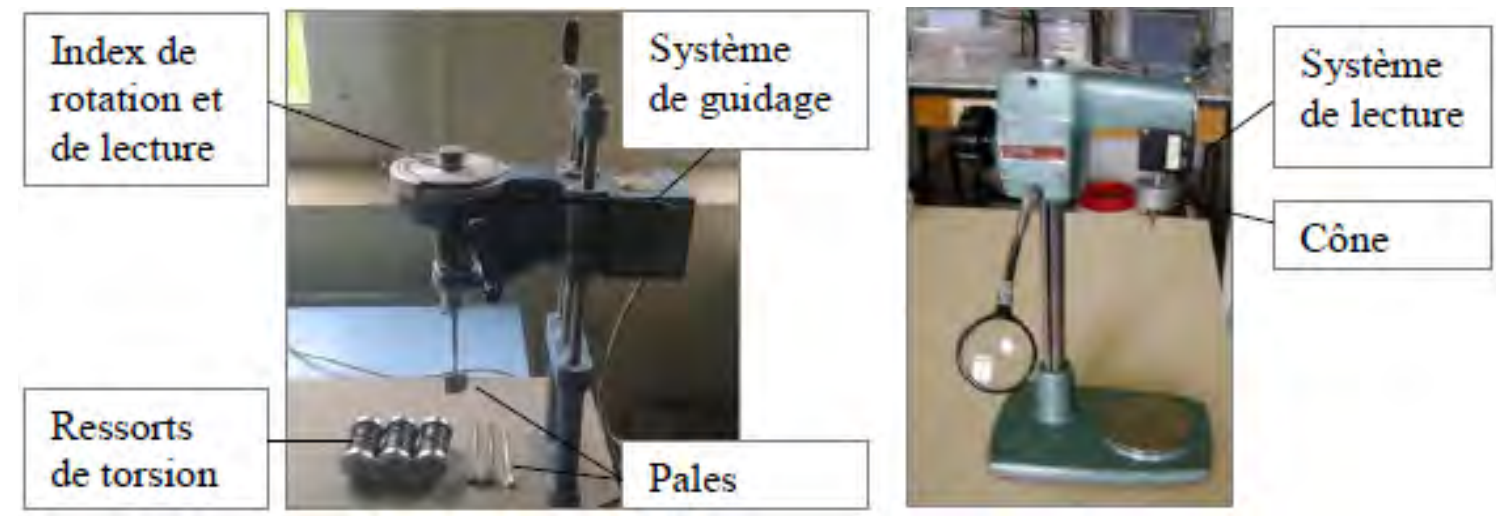

Figure 2. Moyens de mesure de $C_{u}$ : le scissomètre (à gauche) et le fall-cône (à droite). 


\section{XIV èmes Journées Nationales Génie Côtier - Génie Civil \\ Toulon, 29 juin au $1^{\text {er }}$ juillet 2016}

\section{Résultats}

3.1 Évolution de la masse et de la teneur en eau des sédiments durant le séchage

La figure 3 montre la variation de la masse des sédiments portuaires d'Honfleur (KHADRAOUI, 2012) et celle concernant des sédiments de barrage (SANO, 2012). La figure 3a montre en particulier, une comparaison de l'évolution de la masse en fonction de la hauteur initiale de la couche de sédiment lors du séchage.

Les sédiments présentent le même comportement par rapport au temps de séchage, quelle que soit la hauteur initiale des sédiments et l'origine des sédiments.

Deux zones peuvent être différenciées dans ce comportement : une première zone contrôlée par le phénomène de drainage, où la masse du sédiment diminue rapidement, en réduisant de $50 \%$ la teneur en eau initiale pendant les 10 premiers jours de séchage. On observe une deuxième zone où la variation de la masse est de plus en plus faible dans le temps. Dans cette zone, prédomine le phénomène de l'évaporation, responsable de l'apparition de fissures vers le 12ème jour de séchage.

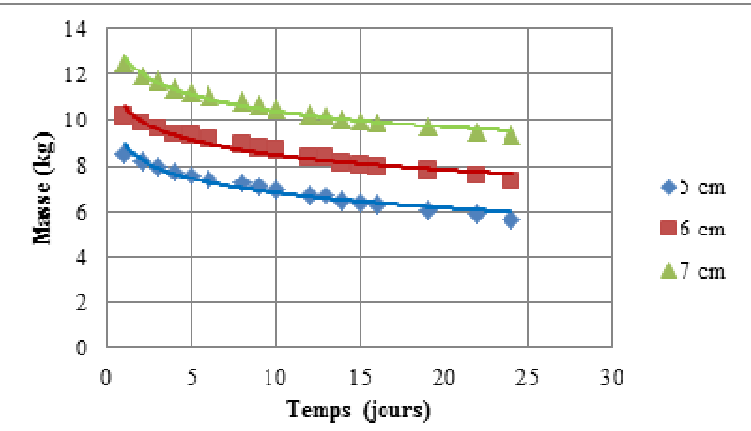

(a) Essais sur les sédiments d'Honfleur Epaisseurs de la couche de sédiments : 5, $67 \mathrm{~cm}$.

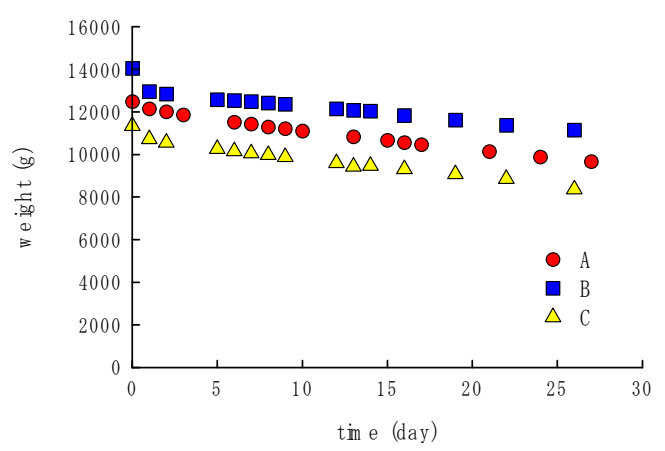

(b) Essais sur les sédiments de barrage A-Isère ; B-Durance ; C-Rhin.

Figure 3. Variation de la masse des sédiments pendant le temps de séchage.

La figure 4 montre la distribution en pourcentages de l'eau des sédiments du port de Cherbourg (DUAN, 2008) durant le temps de séchage et en fonction de la taille du tamis sur lesquels sont déposés les sédiments étudiés. Dans le cas des essais menés par DUAN (2008), trois tailles de tamis avaient été retenues :

$\mathrm{T}_{1}=1.85 \mathrm{~mm}<\mathrm{T}_{2}=2.25 \mathrm{~mm}<\mathrm{T}_{3}=2.80 \mathrm{~mm}$.

Cette figure 4 permet d'illustrer les phénomènes mis en jeu dans les deux zones de séchage. Il est à noter que le point d'inflexion des zones de séchage correspond à la limite de liquidité des sédiments ( $\mathrm{W}_{\mathrm{L}}$ allant de 90 à 100\%). Il n’y a pas d'effet dû à la taille de la maille du tamis sur les vitesses de drainage ou d'évaporation. 


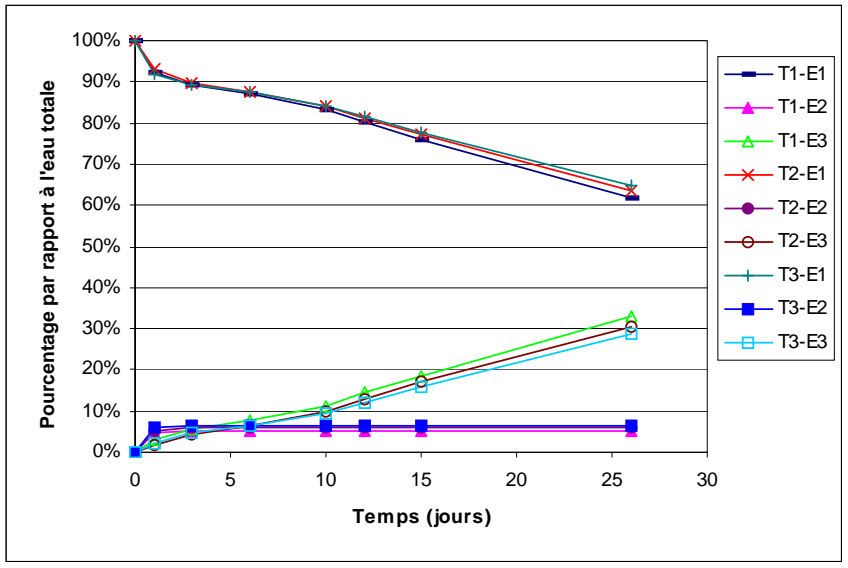

Figure 4. Évolution des pourcentages d'eau drainée, évaporée et contenue dans les sédiments (DUAN, 2008).

3.2 Evolution de la résistance à la cohésion non drainée avec la teneur en eau En ce qui concerne la résistance au cisaillement, les figures 5 et 6 montrent l'évolution de la cohésion non drainée en fonction de la teneur en eau des sédiments du port de Cherbourg (DUAN, 2008) et du port d'Honfleur (KHADRAOUI, 2012).

La figure 5 concerne les résultats obtenus avec le scissomètre et le fall-cône. Cette figure confirme bien que la cohésion non drainée $C_{u}$ est inversement proportionnelle à la teneur en eau du sédiment.

Les différences de résultats obtenus dans le cas des deux essais sont dépendantes de deux paramètres: le type de rupture de chaque essai et la profondeur où l'essai est réalisé. D’une part, l'essai scissométrique est un essai quasi-statique où le cisaillement du sol est pris en compte et considéré comme uniforme. Les mesures sont réalisées à la mi- profondeur de la couche de sédiments. D'autre part, les essais au fall-cône correspondent à un essai dynamique lent et les mesures sont effectuées à la surface du sol.

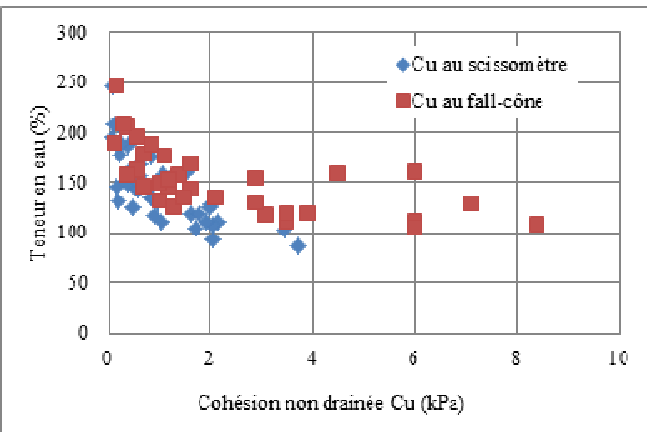

(a) Essais sur les sédiments d'Honfleur

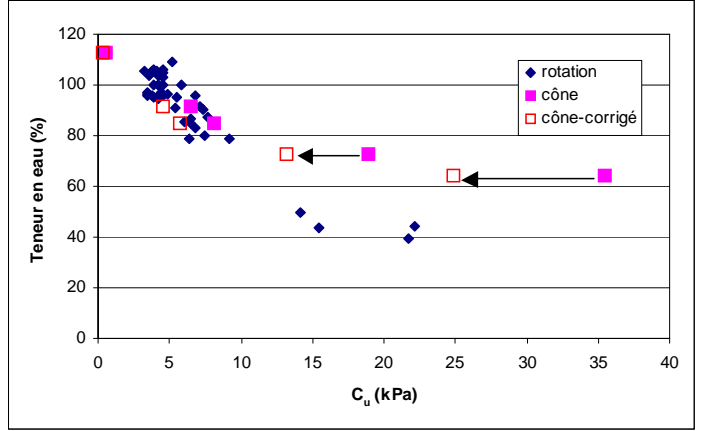

(b) Essais sur les sédiments de Cherbourg

Figure 5. Relation entre $C_{u}-w$.

Les résultats obtenus à partir du fall-cône doivent être multipliés par un coefficient réducteur $\beta$ défini par BJERRUM (1954), lequel est donné sur le tableau 1. 
Tableau 1. Valeur de $\beta$ selon BJERRUM (1954).

\begin{tabular}{ll}
\hline Sols fins & Coefficient $\boldsymbol{\beta}$ \\
\hline Crème de vase, tourbe & 0.6 \\
Vase argileuse & 0.7 \\
Argile vasarde & $0.8-0.9$ \\
Argile & 1.0 \\
\hline
\end{tabular}

Comme les sédiments s'apparentent plus à de la crème de vase pour de faibles consistances ou à de la vase argileuse, les mesures obtenues avec l'essai au fall-cône, ont été multipliées par un coefficient $\beta=0.6$ et 0.7 , les résultats corrigés sont plus concordants. Ils sont donnés à la figure 6 .

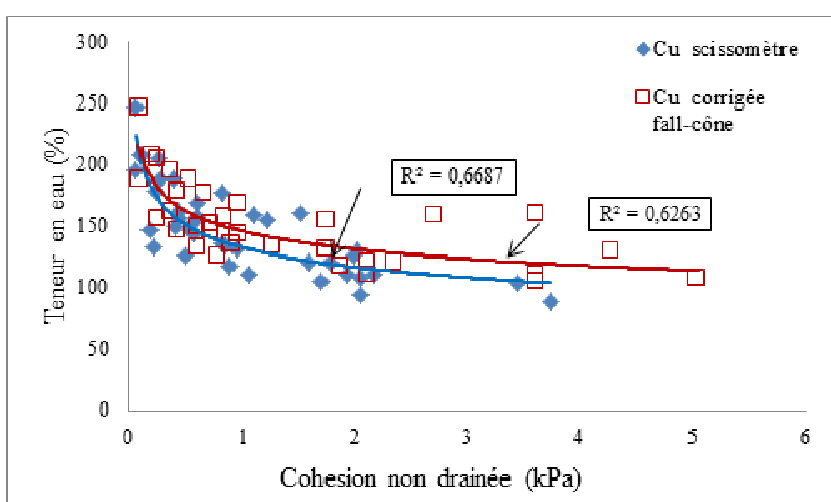

(a) Essais sur les sédiments d'Honfleur

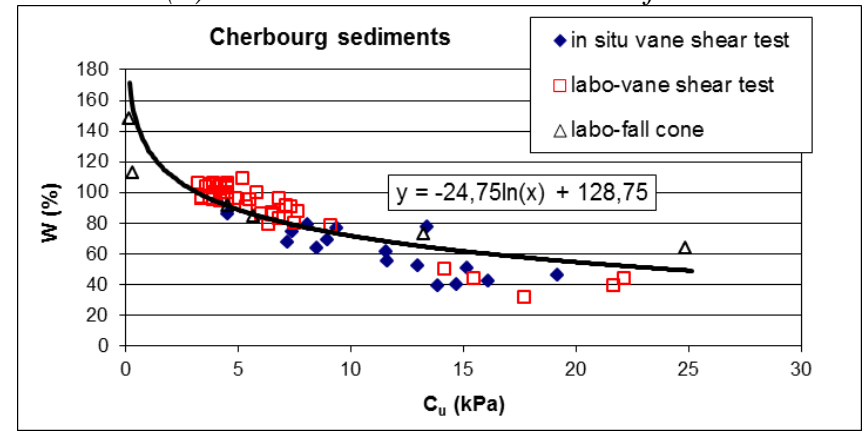

(b) Essais sur les sédiments de Cherbourg

Figure 6. Relation entre $C_{u}$-w obtenue avec le scissomètre et le fall-cône après application du coefficient $\beta$.

\section{Conclusion}

Le séchage naturel dépend du paramètre temps et des propriétés de la matrice sédimentaire (minéralogie, argilosité, taille des particules, ...).

Ce type de déshydratation peut être défini par la relation de la cohésion non drainée teneur en eau " $C_{u}-w$ " d'une part et par la relation du temps de séchage - teneur en eau "w-temps" d'autre part.

En ce qui concerne le temps de séchage naturel des sédiments, on observe a priori deux 
phases de séchage : une première phase dans laquelle prédomine le phénomène de drainage et où la masse du sédiment diminue rapidement. Puis une deuxième phase contrôlée par l'évaporation de l'eau des sédiments, où la variation de la masse est de plus en plus faible voire devenant constante dans le temps. Ces phases de séchage se distinguent par la limite de liquidité des sédiments.

La cohésion non drainée $C_{u}$ peut être étudiée en laboratoire à l'aide des essais utilisant le scissomètre et le fall-cône. Ces essais montrent bien les évolutions de $C_{u}$, cependant ces essais sont régis par des procédures différentes. Cette différence de procédures amène à corriger les valeurs obtenues par le "fall-cône". Elles peuvent être corrigées par les coefficients de réduction de Bjerrum ou bien des coefficients doivent être déduits de ces essais en corrélant les valeurs issues du fall-cône sur celles du scissométre. Par ailleurs on observe bien que la cohésion non drainée est inversement proportionnelle à la teneur en eau du sédiment, l’allure des courbes dépend du sédiment étudié.

\section{Références bibliographiques}

BJERRUM L. (1954), Geotechnical properties of Norwegian marine clays, Geotechnique, Vol. 4(2), pp 49-69. [Also published in Norwegian Geotechnical Institute. Publication 4] http://dx.doi.org/10.1680/geot.1954.4.2.49

DUAN Z. (2008). Caractérisation, stabilisation et solidification de sédiments fins marins. Thèse Université de Caen, 198 p.

EL FADILI M., MESSAGER M. (2015). Enquête dragage 2011. Synthèse des données. Cerema - Cetmef, 39 p.

SANO K. (2012). Application of site investigation technologies to dam sediments. Rapport de Master. Université de Caen et IFSTTAR Nantes, 25 p.

KHADRAOUI S. (2012). Séchage naturel de sédiments. Rapport de Master 1 Université de Caen, 39 p. 